\title{
Subtype determination of Drosophila embryonic external sensory organs by redundant homeo box genes BarH1 and BarH2
}

\author{
Shin-ichi Higashijima, Tatsuo Michiue, Yasufumi Emori, and Kaoru Saigo ${ }^{1}$ \\ Department of Biophysics and Biochemistry, Faculty of Science, University of Tokyo, Bunkyo-ku, Tokyo 113, Japan
}

\begin{abstract}
BarH1 and BarH2 are two closely related homeo box genes that form a small complex at the Bar locus on the $\mathrm{X}$ chromosome of Drosophila. By immunostaining, we showed that BarH1 and BarH2 proteins are coexpressed in cells belonging to the central and peripheral nervous systems in embryos. In external sensory (es) organs, their expression was particularly apparent in thecogens (glial cells) and neurons at late development. Although deletion of $B a r H 2$ caused no appreciable morphological change in es organs, the simultaneous deletion of BarH1 and BarH2 led to a homeotic change in these organs with consequent conversion from campaniform-like sensilla to trichoid sensilla. In contrast, the overexpression of either BarH1 or BarH2 resulted in opposite morphological change. It would thus follow that BarH1 and BarH2 are a pair of redundant homeo box genes required for the subtype specification of es organs.
\end{abstract}

[Key Words: Drosophila melanogaster; sensory organ; Bar homeo box genes; redundant genes; nervous system]

Received February 6, 1992; revised version accepted March 16, 1992.

The peripheral nervous system (PNS) of the Drosophila embryo consists of various sensory organs having precise modes of arrangement (Campos-Ortega and Hartenstein 1985; Ghysen et al. 1986; Bodmer and Jan 1987). In the thoracic and abdominal segments, locations of virtually all PNS neurons have been determined, and the relationship between larval sensory organs and embryonic PNS cells has been clarified (Campos-Ortega and Hartenstein 1985; Dambly-Chaudiere and Ghysen 1986). Sensory organs are of two major groups: external sensory (es) organs, which may function as mechanosensors or chemosensors, and internal chordotonal (ch) organs, which function as possible stretch receptors (Mclver 1985; Zacharuk 1985; Hartenstein 1988). Neurons of either es or ch organs have single dendrites, whereas the third class of neurons in the PNS have multiple dendrites (md) and are not associated with definite sensory structures (Ghysen et al. 1986; Bodmer and Jan 1987). es organs have several subtypes, among which the campaniform-like sensillum, with a papilla, and trichoid sensillum, with a long hair surrounded by a socket, are two major groups in thoracic and abdominal segments (Dambly-Chaudiere and Ghysen 1986; Hartenstein 1988). Irrespective of differences in morphology, they are highly homologous to each other in various respects (Ghysen et al. 1986; Bodmer et al. 1989; Blochlinger et al. 1990).

\footnotetext{
${ }^{1}$ Corresponding author.
}

Genetic analysis of PNS formation has led to the identification of several relevant genes. The cut gene encodes a homeo domain protein, probably acting as a homeotic selector for es-ch determination (Bodmer et al. 1987; Blochlinger et al. 1988, 1991), whereas the absence of the numb gene product with zinc fingers results in conversion of the neuron and thecogen (glial cell) to outer support cells (Uemura et al. 1989). The prospero protein, which is essential for the axonal outgrowth and pathfinding of the PNS and central nervous systems (CNS), has a putative DNA-binding domain similar in sequence to the DNA-recognition helix 3 of the homeo domain (Doe et al. 1991; Vaessin et al. 1991; Matsuzaki et al. 1992|. However, no gene has been found related to the subtype determination of es organs.

$B a r H 1$ and $B a r H 2$ are two closely related homeo box genes that form a small complex at the Bar locus on the X chromosome of Drosophila (Higashijima et al. 1992). Their overexpression at the late third instar causes reduced eye morphology similar to that noted in the $B$ mutation (Kojima et al. 1991; unpubl.), in which BarH1 is duplicated (Sturtevant 1925). BarH1 and BarH2 proteins are not only coexpressed but also functionally required in two photoreceptors, R1 and R6, along with primary pigment cells (Higashijima et al. 1992). In addition to their roles in normal eye development, both genes are known to be extensively expressed during embryogenesis (Koiima et al. 1991; Higashijima et al. 1992). 
In this study the expression and functions of these Bar homeo box genes in the embryo were examined. BarH1 and $\mathrm{BarH2}$ were coexpressed mainly in a subset of neurons and thecogens in the PNS and the CNS. In the PNS, their expression was particularly prominent in es organs. The simultaneous deletion of $\mathrm{BarH} 1$ and $\mathrm{BarH} 2$ resulted in homeotic changes in es organs, that is, the conversion from campaniform-like sensilla to trichoid sensilla, although the deletion of $\mathrm{BarH} 2$ caused no appreciable change in es organs. In contrast, their overexpression had the opposite result. Thus, it is suggested that $\mathrm{BarH} 1$ and $\mathrm{BarH} 2$ are paired, redundant homeo box genes, acting as homeotic selectors for the subtype specification of es organs.

\section{Results}

Coexpression of BarH1 and BarH2 proteins in a limited number of embryonic cells

BarH1 and BarH2, sibling homeo box genes in the Bar region, are mainly expressed during embryogenesis and metamorphosis (Kojima et al. 1991; Higashijima et al. 1992). To examine their temporal and spatial expression patterns in embryos, polyclonal antibodies were generated. Antibody $\mathrm{S} 12$ raised against $\mathrm{BarH1}$ protein could recognize BarH1 and $\mathrm{BarH} 2$ proteins (Fig. 1A, lanes 1,2), whereas the $\mathrm{Y} 2$ antibody raised against the $\mathrm{BarH} 2$ protein was BarH2-specific (lanes 5,6). The BarH1-specific antibody S1-1 (lanes 3,4$)$ was generated by absorption of the S12 antibody with extracts of Escherichia coli cells producing the $\mathrm{BarH} 2$ protein.

Figure 1, B-G, shows immunostaining patterns of wild-type embryos at 12-14 hr of development. Staining with the three antibodies was virtually the same in all cases. Segmentally repeated, isolated signals, intersegmental stripes of the dorsal region, and intensively stained clusters in the anterior one-third could always be recognized. Note that the $\mathrm{BarH}^{-}$embryo (see below) can be stained by the S1-1 antibody (Fig. 1I) but cannot be stained by the Y2 antibody at all (Fig. 1J). Close examination indicated that segmental patterns were classifiable into four types, representing $\mathrm{T} 1, \mathrm{~T} 2-\mathrm{T} 3, \mathrm{Al}-\mathrm{A} 7$, and A8-telson. No significant differences in signal positions and intensity in the staining patterns with the three antibodies could be found in the case of the segmental patterns (E-G for lateral signals in an abdominal segment).

Anterior signal-clusters, located at various focal planes in Figure 1, were found to be near the surface of embryos at earlier stages (Fig. 2). During late embryogenesis, anterior signals change their locations considerably owing to head involution (Campos-Ortega and Hartenstein 1985). BarH1 and/or BarH2 proteins began to express in anterior segments, labium, maxilla, and procephalic lobe at 5.5-6.5 hr of development (Fig. 2A). In 6.5- to $7.5-\mathrm{hr}$ embryos, S12-antibody staining reached its maximum level and other stained regions could be seen in mandible, clypeolabrum, hypopharynx, and procephalic lobe (Fig. 2B). The staining patterns by S1-1 and Y2 antibodies

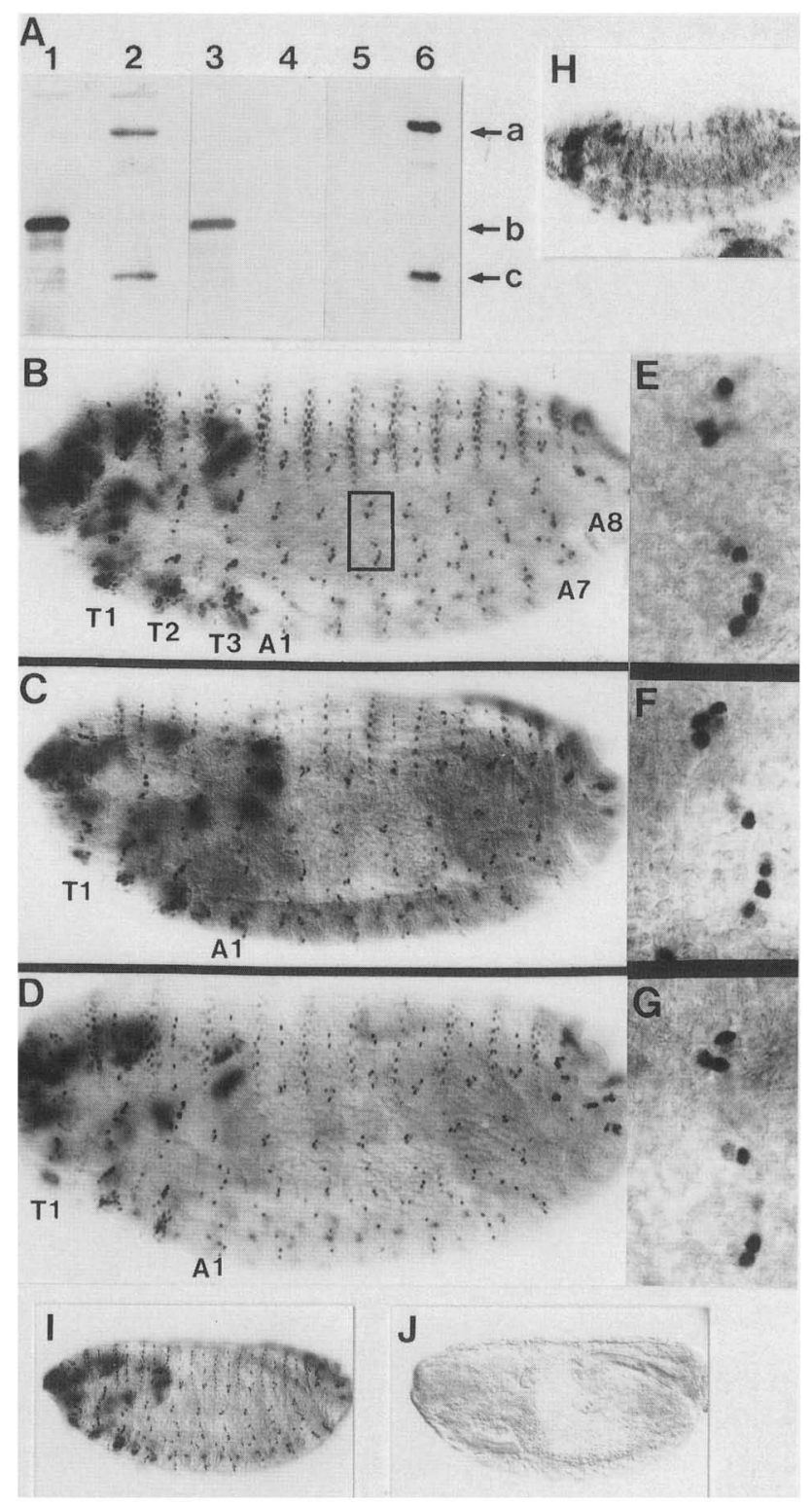

Figure 1. (A) Immunoblotting profiles of the S12 (lanes 1,2), S1-1 (lanes 3,4), and Y2 (lanes 5,6) antibodies. (Lanes 1,3,5) Extracts of $E$. coli cells producing the BarH1 fusion protein; (lanes $2,4,6)$ extracts of $E$. coli cells producing the BarH2 fusion protein. (Arrows a, b, and c) Locations of the $\mathrm{BarH} 2$ fusion protein (molecular weight 60,000 ), BarH1 fusion protein (molecular weight 42,000$)$, and a degradation product of the $\mathrm{BarH2}$ fusion protein (molecular weight 35,000 ), respectively. $(B-D)$ Immunostaining patterns of 12- to 14-hr embryos with S12 (B), S1-1 $(C)$, and $Y 2(D)$ antibodies. (E-G) High-magnification pictures of abdominal lateral signals in $B-D$. The region enclosed by a box was enlarged in the case of $B$. $(H)$ In situ hybridization pattern of an 11-to 12-hr embryo with the BarH1-specific antisense RNA. Anterior is left; dorsal is up. (T1-T3) Thoracic segments; (A1, A7, A8) abdominal segments. (I) Immunostaining patterns of the BarH2 $2^{-}$embryo $(D f(1 \mid B H 2)$ with S1-1. (J) Immunostaining patterns of the $D f(1) B H 2$ embryo with Y2.

were quite the same (data not shown). $\mathrm{BarH} 1$ and $\mathrm{BarH} 2$ would thus appear to be coexpressed in a particular set of 


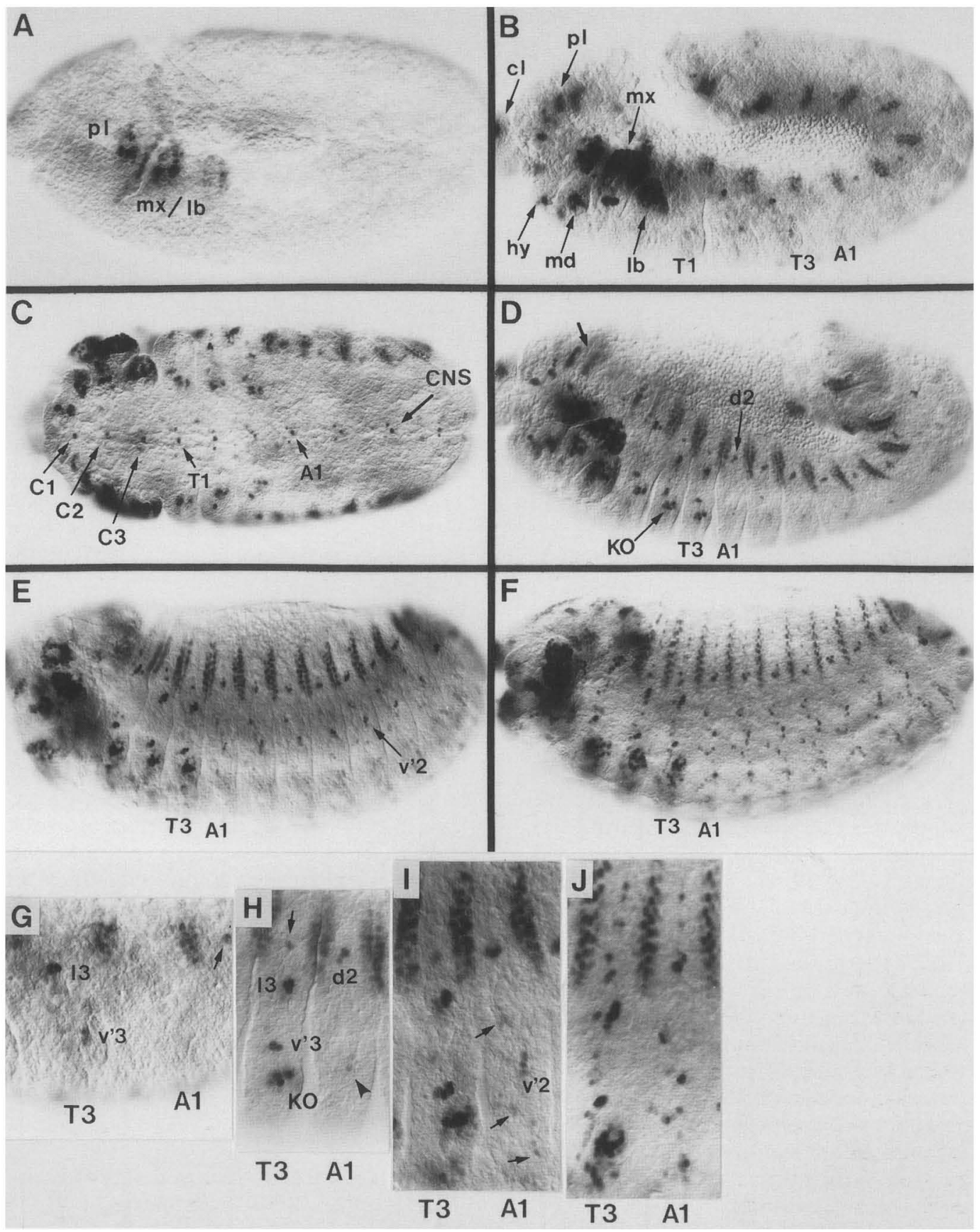

Figure 2. Developmental profiles of $\mathrm{BarH1} / \mathrm{BarH2}$ expression at $25^{\circ} \mathrm{C}$. Anterior is left; except for $C$, dorsal is up. (A) A 5.5- to 6.5-hr embryo (lateral view); $(B)$ 6.5- to 7.5-hr embryo (lateral view); $(C)$ 7- to 8-hr embryo (ventral view); $(D)$ 8- to 9-hr embryo (lateral view); (E) 9.5- to 10.5-hr embryo (lateral view); $(F)$ 11- to 12 -hr embryo (lateral view); $(G-I)$ enlargements of $B-F$, respectively. The arrowhead in $H$ indicates signals probably corresponding to v'es2. Arrows in $G-I$ show signals possibly due to simple es organs. (pl) Procephalic lobe; (mx) maxilla; (lb) labium; (cl) clypeolabrum; (hy) hypopharynx; (md) mandible; (T1) first thoracic segment; (T3) third thoracic segment; (Al) first abdominal segment $;(\mathrm{Cl}-\mathrm{C} 3)$ three gnathal segments; $(\mathrm{KO})$ Keilin's organ. Subtypes of es cells: $(\mathrm{d} 2)$ des2; $\left(\mathrm{v}^{\prime} 2\right) \mathrm{v}^{\prime} \mathrm{es} 2$; (v'3) v'es3; (13) les3 (see Fig. 3 for details).

cells even in anterior signal clusters. Because of this strict coexpression, only staining patterns produced by the $\mathrm{S} 12$ antibody appear in the following sections. The results in most cases could be confirmed by staining with S1-1 and Y2.
Expression of BarH1/BarH2 proteins in the CNS and PNS

Except for stripes of the dorsal region, BarH1/BarH2positive signals appeared distributed in parallel with the 
CNS and PNS. To find a possible relation between BarH1/BarH2 expression and nervous systems, 12- to 14-hr embryos were examined by double staining with the $S 12$ antibody and the neuron-specific monoclonal antibody, mAb22C10 (Fuiita et al. 1982; Zipursky et al. 1984), which stains both the cytoplasm and membrane so that axons and dendrites can be traced easily (Fig. $3 \mathrm{~B}, \mathrm{~F})$. In thoracic and abdominal repeats, strong and weak $\mathrm{BarH1/BarH2-positive} \mathrm{signals} \mathrm{had} \mathrm{a} \mathrm{tendency} \mathrm{to}$ make pairs (see Fig. 3C,G); but owing to technical difficulties, only stronger signals could be reproducibly detected as black dots upon double staining (Fig. 3B,F).

Figure 3, B and F, respectively, shows the staining patterns of two thoracic and two abdominal segments. A comparison of these patterns with previously characterized maps of neurons in PNS in Figure 3, A and E, indicated that BarH1/BarH2-positive signals in the thoracic and abdominal segments were intimately related to neurons belonging to es organs. Cells related to ch organs and md neurons showed no staining. Pictures of lesscrowded regions indicated that stronger signals corresponded to the nucleus of the cell directly associating with an es neuron (arrow a in Fig. 3F), whereas weaker ones corresponded to the nuclei of es neurons. (arrow b in Fig. 3F). The simplest es organ, representative of most es organs in thoracic and abdominal segments, consists of a neuron and a set of three support cells (see Fig. 7, below|. The inner support cell, the thecogen, forms a sheath around the tip of a dendrite, whereas the outer support cells, the trichogen and tormogen, secrete cuticle structures of the sensillum. BarH1/BarH2-expressing cells failed to be stained by anti-lac $Z$ antibody in an enhancer trap line, A1-2nd-29, in which lac $Z$ was expressed only in the trichogen and tormogen (Fig. 4A-D; Hartenstein and Posakony 1990); BarH1/BarH2-positive es cells were stained with the anti-lac $Z$ antibody in another enhancer trap line, Q6, in which the neuralized gene is disrupted by $P$ insertion and all four es cells express the lacZ protein (S. Higashijima, unpubl.). Thus, es cells strongly expressing $\mathrm{BarH} 1$ and $\mathrm{BarH} 2$ proteins are concluded to be glial thecogen cells. However, in complex es organs such as basiconical sensilla and Keilin's organs in thoracic segments, some fractions of outer support cells expressed $\mathrm{BarH} 1 / \mathrm{BarH} 2$ proteins /arrow $\mathrm{c}$ in Fig. 3B for a basiconical sensillum).
Figure 4, H and I, respectively, shows patterns of some anterior clusters and those near the posterior end. The most prominent $\mathrm{BarH1/BarH2-positive} \mathrm{cluster} \mathrm{in} \mathrm{the}$ anterior region corresponded to the antennomaxillary complex, the largest sensory organ in the embryo and larva, where nearly all es neurons and their support cells apparently expressed BarH1/BarH2 proteins. Although no detailed analysis was conducted, other sensory organs from labium, hypopharynx, and clypeolabrum appeared to be positively stained with the anti-BarH1/BarH2 antibody. All complex es organs situated at the posterior terminal appeared to include BarH1/BarH2-positive, esrelated cells (Fig. 4I).

Figure $4 \mathrm{H}$ also shows that the brain /supraesophageal ganglion) contained a few BarH1/BarH2-positive clusters, most likely derivatives of $\mathrm{BarH1/BarH2-positive}$ clusters located medially in the procephalic lobe (thick arrow in Fig. 2D). The expression of $\mathrm{BarH} 1$ and $\mathrm{BarH} 2$ proteins was also observed in the ventral ganglion, where BarH1/BarH2-positive signals formed segmentally repeated patterns as in the case of PNS (Fig. 4F). In addition to thoracic and abdominal segments, three gnathal segments, Cl-C3, were BarH1/BarH2-positive as evident from Figure 2C. Although a detailed analysis was not carried out, locations of most BarH1/BarH2 protein-expressing cells, which did not express the engrailed gene (Fig. 4G), were restricted to the ventral surface of the ganglion.

Cells corresponding to dorsal, intersegmental stripes (Figs. 1 and 2) have yet to be shown on any embryonic fate map. However, the coincidence of their locations with those of dorsal cuticle hairs in the anterior and/or posterior regions of each segment in larva would suggest that cells forming these 11 stripes may correspond to the primodia of two of the three types of dorsal hairs (Campos-Ortega and Hartenstein 1985).

In summary, except for intersegmental, dorsal epidermis cells, only es neurons and a fraction of their support cells in PNS along with some CNS cells produce BarH1 and $\mathrm{BarH} 2$ proteins in the embryo.

\section{Expression of $\mathrm{BarH} 1 / \mathrm{BarH} 2 \mathrm{mRNA}$}

Whole-mount in situ hybridization was carried out using BarH1- and BarH2-specific antisense RNA probes. The

Figure 3. BarH1/BarH2 expression in the PNS of 12 - to 14 -hr embryos. Anterior is left; dorsal is up. $(A-D)$ Thoracic segments T2 and T3. $(E-H)$ Abdominal segments A1 and A2. Locations of es neurons were determined based on the results of Dambly-Chaudiere and Ghysen (1986) and Blochlinger et al. (1990). $(B, F)$ Double staining with S12 (black signal) and mAb22C10 (brown signal) antibodies. Explanations are shown schematically in $A$ and $E$. (Red cells) es neurons; (blue circles) nuclei of the putative es thecogens; (green circles/nuclei of the putative outer support cells that exceptionally express BarH1/BarH2 proteins; (purple circles) nuclei of es neurons strongly stained with the $\mathrm{S} 12$ antibody. Location and number of thecogens are unclear in the case of the Keilin's organ (KO). (Arrows $\mathrm{a}$ and b) Nuclei of a putative lesC thecogen and lesB neuron, respectively, (arrow c) one of the nuclei of outer support cells of the les3 (basiconical sensillum). $(C, D, G, H) \mathrm{S} 12$ staining patterns of es neurons and thecogens. A part of $C$ was replaced by the counterpart of another embryo for clarity. $(D, H)$ Signal intensities in nuclei of es neurons (red circles), along with structural features of corresponding sensilla, are shown schematically. (VW) Very weak; (W) weak; (M) moderate. Blue circles correspond to nuclei of thecogens, in which $\mathrm{BarH1}$ and BarH2 proteins are strongly expressed. "Complex" es organs (les3, v'es3, and KO in the thoracic segment, and des2 and $\mathrm{v}^{\prime}$ es 2 in the abdominal segment) were not analyzed. Abdominal desB and thoracic $v^{\prime} e s A / v^{\prime} e s B$ could not be analyzed because of their close proximity. 

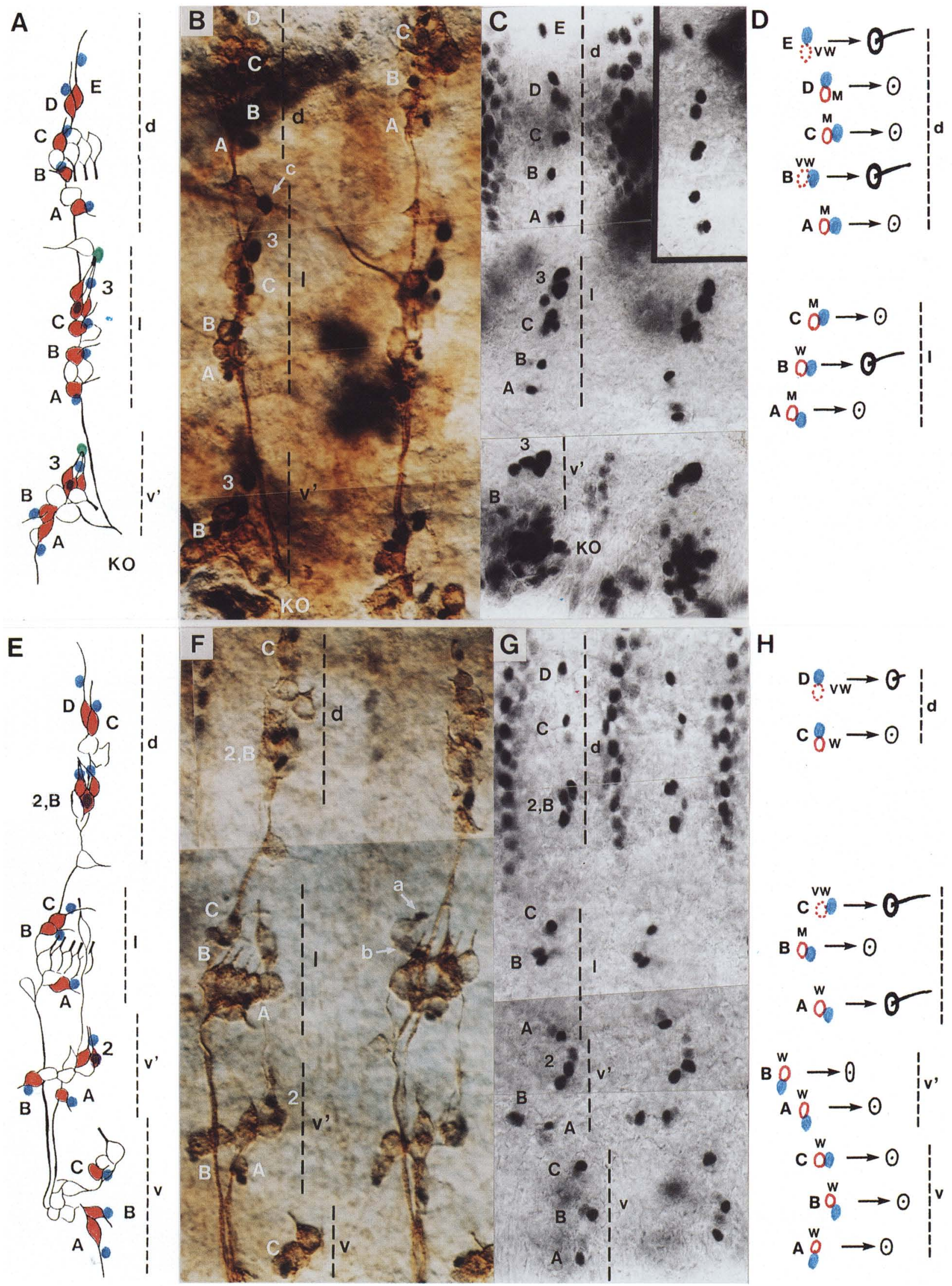

Figure 3. (See tacing page for legend.) 
expression of mRNA was found to be virtually the same as that of proteins, thus confirming the nervous systemspecific expression of $\mathrm{BarH} 1$ (Fig. $1 \mathrm{H}$ ) and BarH2 (data not shown).

\section{Requirement of $\mathrm{BarH1/BarH} 2$ proteins} for es organ development

BarH1 and BarH2 were found to be quite similar in structure (Higashijima et al. 1992) and expression (Fig. 1), and thus could reasonably be expected to be a pair of redundant homeo box genes. The inactivation of either may cause no distinctive change in phenotype, whereas significant phenotypic changes may be induced by their simultaneous deficiency. An attempt was thus made to determine the possible roles of $\mathrm{BarH} 1 / \mathrm{BarH} 2$ proteins in es organ development using a deficiency chromosome $D f(1) B^{263-20}$ (Sutton 1943), which lacked a 210-kb-long region that included both $\mathrm{BarH1}$ and $\mathrm{BarH2}$ (Fig. 5A; Higashijima et al. 1992). $D f(1) B^{263-20}$ is not embryonic lethal, but the individual hemizygous for this deficiency dies in an early larval stage (see Materials and methods). It is thus possible to isolate and examine $\mathrm{Bar}^{-}$first-instar larvae using yellow marker (for details, see Materials and methods). The PNS of the mutant embryo at 12-14 hr of development was initially examined using several markers. No defects could be detected when visualized by mAb22C10 (Fig. 6A-C) or anti-HRP (Jan and Jan 1982), another neuron-specific antibody (data not shown). Axon projections and dendrite innervations thus cannot be considered abnormal. Outer support cells of 10- to 14-hr embryos also appeared normal with respect to locations and shape, as visualized by the anti-lacZ antibody against an A1-2nd-29 background (Fig. 4E). The absence of BarH1/BarH2 should thus have little or no effect on PNS development at least up to the 12-14 hr embryonic stage, although possible change in glial cells cannot be eliminated because of lack of a suitable glialcell marker. As schematically shown in Figure 7, BarH1/ BarH2-expressing cells also produce prospero and cut proteins. Because the absence of these proteins results in morphological changes of es neurons (Bodmer et al. 1987; Doe et al. 1991; Vaessin et al. 1991), it is suggested that their expression is not under the control of BarH1 and/or BarH2.

By scanning electron microscopy and Nomarski optics, es structures of the first-instar larvae were examined. Figures 6 and 8 show many changes in cuticle structures. In the antenna part of the antennomaxillary complex, a typical dome structure was deformed and had a single dome with two humps or a pair of small domes (arrowheads labeled An in Fig. 6D,E). In the maxillary part of the same complex, two large sensory clusters were seen situated apart from each other (arrowheads labeled $\mathrm{Mx}$ in Fig. 6D,E). One of three hairs, found in a wild-type Keilin's organ (Fig. 6F), disappeared completely, and another was extensively reduced in size (Fig. 6G). The shape of basiconical sensilla, the second class of complex es organs in thoracic segments, was changed from a dome-like to a trichoid-like structure (Fig. $6 \mathrm{H}, \mathrm{I}$ ).
Two types of simple es organs are distributed in thoracic and abdominal segments in wild-type larvae (Dambly-Chaudiere and Ghysen 1986; Bodmer and Jan 1987; Hartenstein 1988). Some simple es organs showed affected morphology in $D f(1) B^{263-20}$. The wild-type embryo has two bristles and two papillae on the lateral region of each abdominal segment (A1-A7) (Fig. 6L). The former correspond to trichoid sensilla innervated by lesA and lesC neurons; the latter campaniform-like sensilla are innervated by lesB and v'es 2 neurons. Note that the $\mathrm{v}^{\prime}$ es2 sensillum is doubly innervated. In the $\mathrm{Bar}^{-} \mathrm{mu}-$ tant, structural changes were apparent in two campaniform-like sensilla, whereas little or no change could be detected in trichoid sensilla (Fig. 6M). In mutant v'es2 and lesB organs, tormogens secreted structures similar to sockets of trichoid sensilla, and trichogens protruded hairs, though relatively short, instead of papillae. The mutant-type lesB hair, somewhat longer than that of $\mathrm{v}^{\prime}$ es2, was one-third as long as the wild-type hair.

Figure $8, A$ and $B$, shows dorsal and lateral clusters of es organs in the thoracic segment of $D f(1) B^{263-20}$ and wild-type larvae, respectively. As with abdominal lateral sensilla, three trichoid sensilla, desE, desB, and lesB, were not affected by the $\mathrm{Bar}^{-}$mutation, whereas all campaniform-like sensilla (desC, desA, lesC, and lesA), except for des $\mathrm{D}$, were morphologically changed to trichoid-type sensilla. The degree of morphological change varied considerably according to the organ. In $\operatorname{des} A$, the change in socket structure was noted to be almost complete, but the extent of bristle change was very slight. In desC, an incomplete socket with very short hair was found. Unlike other campaniform-like sensilla, desD was hardly changed in morphology. lesA and lesC had relatively long hairs, but scanning electron micrographs indicated incomplete change in socket structure (Fig. 6K).

Deficiency of the entire $B$ ar region would thus appear to give rise not only to various morphological changes in complex es organs but to partial conversion as well of simple es organs from campaniform-like sensilla to trichoid sensilla without the neuronal network being affected.

Reverse morphological change by ectopic expression of BarH1 and BarH2

Assuming the absence of Bar homeo box genes to result in conversion from campaniform-like sensilla to trichoid sensilla in simple es organs of the $\mathrm{Bar}^{-}$mutant, reverse morphological change should thus occur by the overexpression of either BarH1 or BarH2 or both. For confirmation of this point, transgenic flies with the BarH1 minigene associated with the heat shock promoter were examined (Kojima et al. 1991). With heat treatment at $37^{\circ} \mathrm{C}$ for $15 \mathrm{~min}$ in 10 - to 11 -hr embryos, significant reverse morphological change occurred. Figure $8 \mathrm{C}$ shows almost complete conversion from trichoid to campaniform-like sensilla in thoracic desE, lesB, and partial change in thoracic desB. No appreciable morphological change was de- 


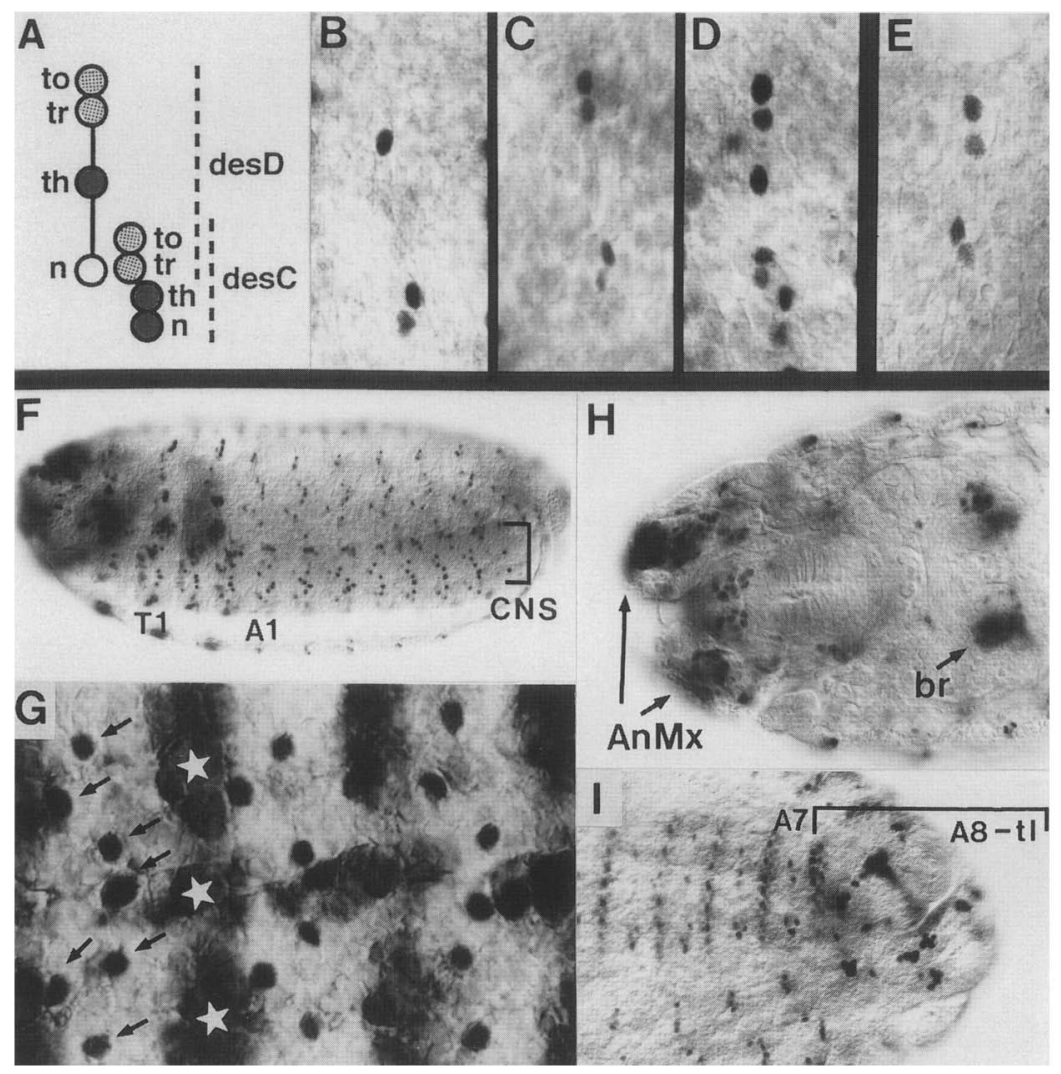

Figure 4. Expression of $\mathrm{BarH} 1 / \mathrm{BarH} 2$ proteins in simple es organs $(A-E)$, complex es organs $(H, I)$, and the CNS $(F, G)$. (A) Relative positions of eight cells belonging to abdominal desC and desD organs. (n) Neuron; (th) thecogen; (tr) trichogen; (to) tormogen. (Solid circles) BarH1/ BarH2-positive cell nuclei; (stippled circles) lacZ-positive cell nuclei. Staining patterns of embryonic abdominal desC and desD cells of an ehhancer-trap line, Al-2nd-29, with the S12 (B) and anti-lacZ $(C)$ antibodies, and a mixture of the $\mathrm{S} 12$ and anti-lac $Z$ antibodies $(D)$. The $l a c Z$ gene is expressed in two outer support cells of each es organ of the Al-2nd-29 embryo. Note that the expression of $\mathrm{BarH1} / \mathrm{BarH} 2$ proteins in the desD neuron is almost negligible. $(E)$ AntilacZ staining of Al-2nd-29 desC and desD cells under a $D f(1) B^{263-20}$ background. Mutant (hemizygous/embryos were identified as the ones unstained with the S12 antibody. $(F)$ Ventral view of a 12- to 14-hr embryo stained with the S12 antibody. $(G)$ Ventral view of a dissected nerve cord derived from a ryxho25 embryo doublestained with the $\mathrm{S} 12$ and anti-lac $Z$ antibodies. In ryxho25, lac $Z$ is expressed in the cytoplasm of engrailed-expressing cells (Hama et al. 1990). Three abdominal segments are shown. Arrows indicate $\mathrm{BarH1/BarH2}$-positive cell nuclei; white stars show en-expressing cells. $(H)$ Dorsal view of anterior BarH1/BarH2-positive clusters. (AnMx) Antennomaxillary complex; (br) brain (supraesophageal ganglion). (I) Dorsolateral view of the posterior half of a 12- to 14-hr embryo stained with the S12 antibody. \{tl| Telson.

tected in campaniform-like sensilla or complex es organs such as Keilin's organs and basiconical sensilla.

The extent of this reverse change in morphology was shown to depend on the time of heat treatment. Little reverse change occurred before $9 \mathrm{hr}$. Maximum effect was noted at 10-11 hr. There was only partial change at $\sim 12 \mathrm{hr}$. That the expression of BarH1/BarH2 proteins in simple es organs becomes prominent in $10-$ to $11-\mathrm{hr}$ em- bryos (see below) appears to be a significant finding. The effect of the overexpression of the $\mathrm{BarH} 2$ gene was also examined by a similar strategy. As shown in Figure 8D, conversion from trichoid to campaniform-like sensilla, similar to that in the BarH1 overexpression, was observed. BarH1 and BarH2 may thus have almost the same functions in the morphogenesis of es organs.

Reverse morphological change may require endoge-

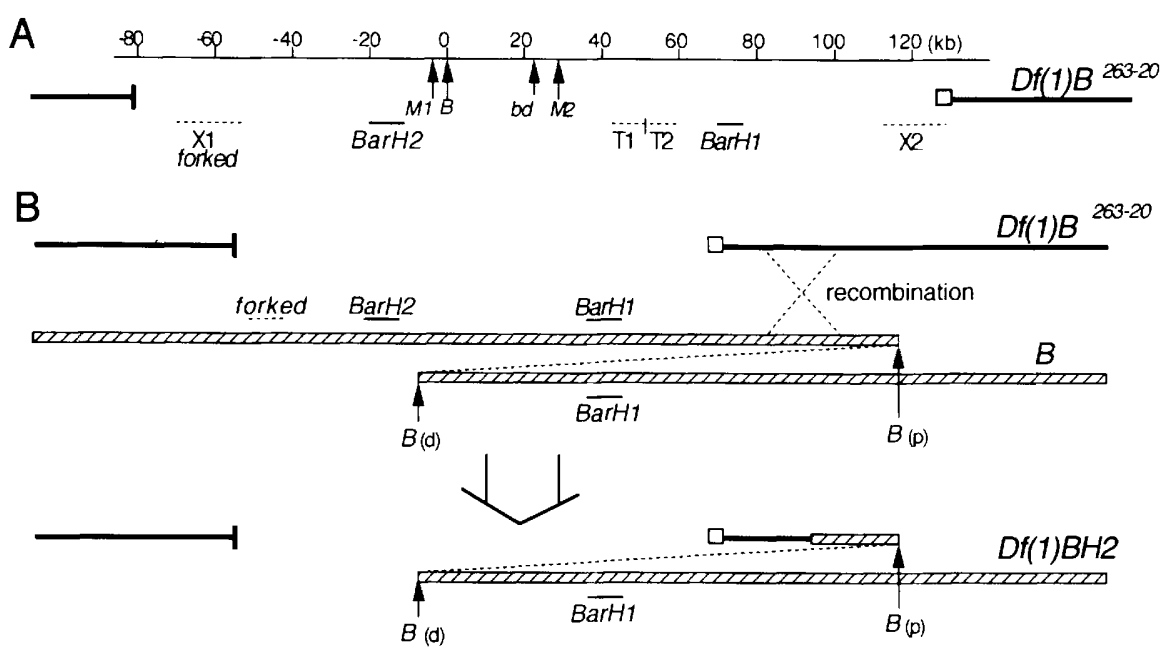

Figure 5. (A) A physical map of the Bar region. An $\sim 210$-kb-long region (from -80 to 130 on the map) is deleted in $D f(1) B^{263-20}$ (Higashijima et al. 1992). Sizes and locations of BarH1 and BarH2 are indicated by horizontal solid lines; dotted lines exhibit those of XI (forked), $\mathrm{X} 2$, and a putative transposon, $\mathrm{T} 1 / \mathrm{T} 2$ (Higashijima et al. 1992). Arrows labeled $M 1, B, b d$, and $M 2$ indicate the positions of $B a r$ breakpoints in $B^{M I}, B, B^{b d}$ and $B^{M 2}$ mutants, respectively (Tsubota et al. 1989; Higashijima et al. 1992). (B) Construction of a BarH2 null mutant, $D f(1) B H 2 . B(\mathrm{p})$ and $B(\mathrm{~d})$ show the proximal end of the first $B$ repeat and distal end of the second $B$ repeat, respectively. 

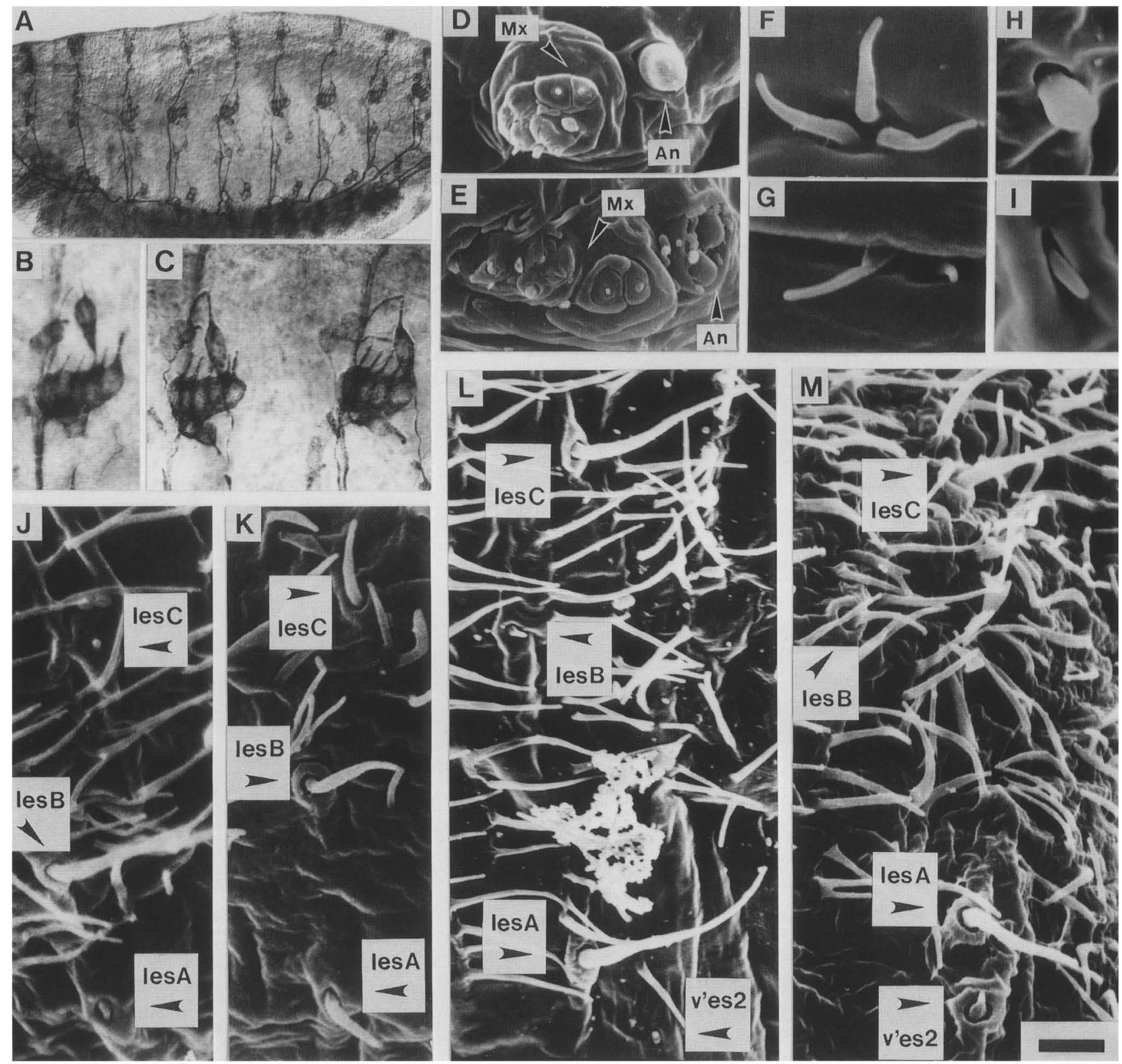

Figure 6. (A) Whole-mount mAb22C10-staining pattern of a 12- to 14-hr $D f(1) B^{263-20}$ embryo. Enlarged figures of two abdominal clusters are shown in C. $B$ exhibits a wild-type lateral cluster. Mutant (hemizygous) embryos were identified as those unstained with the S12 antibody upon double staining. $(D-M)$ Scanning electron micrographs of various es organs. $(D, E)$ Antennomaxillary complexes of wild-type and $D f(1) B^{2630-20}$ first-instar larvae, respectively. (An) Antenna; $(M x)$ maxillary complex. $(F)$ Wild-type Keilin's organ; $(G)$ $D f(1) B^{263-20}$ Keilin's organ; $(H)$ wild-type basiconical sensillum; $(I) D f(1) B^{263-20}$ basiconical sensillum; $(/)$ thoracic es organs (wild type); $(K)$ thoracic es organs $\left[D f(1) B^{263-20}\right] ;(L)$ abdominal es organs (wild type); $(M)$ abdominal es organs $\left[D f(1) B^{263.20}\right]$. Partial deformation of bristle structures in $K$ and $M$ are the result of the deficiency of the forked gene in $D f(1) B^{263-20}$ (see Fig. 5A). Bar, $5 \mu \mathrm{m}$ in $D$ and $E, 1.7$ $\mu \mathrm{m}$ in $F-I$, and $3 \mu \mathrm{m}$ in $I-M$.

nous BarH1 and/or BarH2 genes, which may be activated by overexpressed exogenous BarH1 or BarH2. For clarification of this point, examination was made as to whether there was reverse morphological change under a $D f(1) B^{263-20}$ background. Similar conversion from trichoid to campaniform-like sensilla was noted following the heat induction of BarH1 at $\sim 11$ hr (data not shown). However, not only intrinsic trichoid sensilla but $\mathrm{Bar}^{-}$. dependent trichoid-like structures as well were found to be converted to campaniform-like sensilla. Reverse morphological change thus requires no endogenous BarH1 or BarH2. In contrast to simple es organs, no appreciable recovery of morphology could be induced by $\mathrm{BarH} 1$ overproduction in complex es organs, in which the BarH1/ BarH2 expression occurs at a much earlier stage.

From these observations, BarH1 and BarH2 were con- 

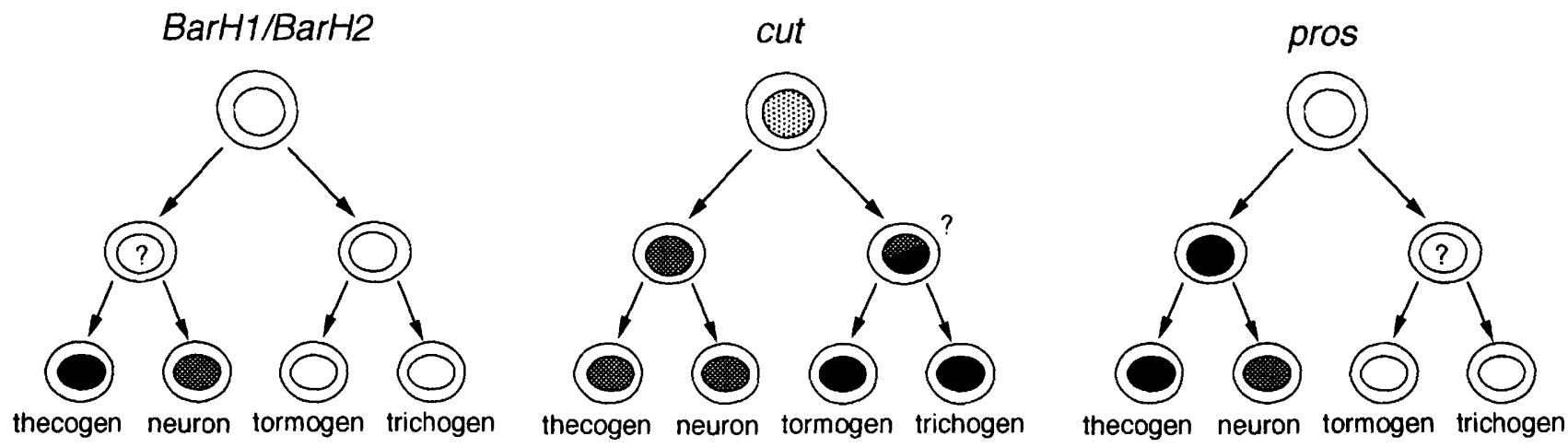

Figure 7. Expression patterns of BarH1/BarH2, cut, and prospero (pros) proteins in nuclei of simple es organ cells. Stippled areas show weak expression; solid areas show strong expression. Expression patterns of cut and prospero proteins were examined by Blochlinger et al. (1990) and Vaessin et al. (1991), respectively.

cluded to be the paired homeotic genes required for the fate determination of es organs. Their presence promotes the induction of campaniform-like structures, whereas their absence facilitates trichoid-like structure formation.

\section{Mutual redundancy of $\mathrm{BarH1}$ and $\mathrm{BarH} 2$}

So far, neither viable mutations affecting the structure and/or function of sensory organs nor lethal point mutations have been mapped in the Bar region, where both $\mathrm{BarH} 1$ and $\mathrm{BarH} 2$ are located. Our previous attempt to isolate the lethal mutation in this region was not successful. About $2300 \mathrm{X}$-linked lethal mutations were examined, but none could be found within the deleted region in $D f(1) B^{263-20}$. A BarH2 null mutation could, however, be generated by an unequal meiotic recombination between $B$, having a BarH1 duplication, and $D f(1) B^{263-2 O}$ chromosomes (Fig. 5B). In contrast to $D f(1) B^{263-20}$ associated with a larval lethal mutation, the resultant $D f(1) B H 2$ flies (forked ${ }^{-} \mathrm{BarH2}^{-} \mathrm{BarH1}{ }^{+}$) were fully viable and possessed almost normal eyes (data not shown).

Using Nomarski optics, the morphology of larval sensilla of this mutant was examined. No significant difference in sensillum structure of larvae could be found between this mutant and wild type, thus, virtually confirming the notion that $\mathrm{BarH} 1$ and $\mathrm{BarH} 2$ are a pair of homeo box genes that determine the morphology of the es organs of embryos in a completely redundant manner.

\section{Intimate relation between $\mathrm{BarH} 1 / \mathrm{BarH} 2$ protein content in es neurons and trichoid campaniform-like sensillum selection in wild-type es organs}

BarH1/BarH2 proteins are expressed in both neurons and thecogens in two types of simple es organs, campaniform-like and trichoid sensilla. The expression levels of BarH1/BarH2 proteins and morphological features of each es organ are summarized in Figure $3, \mathrm{D}$ and $\mathrm{H}$. Strong expression of $\mathrm{BarH1/BarH2}$ proteins was observed in all thecogens regardless of sensillum type, whereas that in neurons varied according to the organ.
Except for abdominal desD, all campaniform-like sensilla appeared to have neurons expressing $\mathrm{BarH} 1 / \mathrm{BarH} 2$ at a relatively high level ( $\mathrm{M}$ or $\mathrm{W})$. The $\mathrm{BarH1/BarH2}$ expression in neuron of trichoid sensilla was either null or at a very low level (VW). Although abdominal desD, whose BarH1/BarH2 expression is almost null (Fig. 3G), has been assigned as a campaniform-like sensillum (Campos-Ortega and Hartenstein 1985; DamblyChaudiere and Ghysen 1986), our observations indicated that it had a bristle about one-quarter as long as that of an authentic trichoid (Fig. 8E,F), so that abdominal desD may rather be a hybrid between trichoid and campaniform-like sensilla. The labeling intensity of abdominal lesA (trichoid) is relatively high (W), but reproducibly much weaker than that of the neighboring campaniformlike sensillum, lesB (Fig. 3G).

Based on the discussion above, it is suggested that the amounts of BarH1/BarH2 proteins in neurons are an important factor determining subtype in simple es organs.

\section{Developmental profiles of $\mathrm{BarH} 1 / \mathrm{BarH} 2$}

protein expression in simple es organs

Analysis of DNA replication patterns indicates that each es organ originates from a single ectodermal precursor cell, from which four es cells are produced in a lineagedependent manner (Bodmer et al. 1989). Cells forming a multiple-innervated es organ are also thought to be related by lineage. Developmental stage dependency of $\mathrm{BarH1} / \mathrm{BarH2}$ expression in the gnathocephalic region is described in a previous section. The first signals of $\mathrm{BarH1} / \mathrm{BarH} 2$ expression in thoracic and abdominal segments appeared in embryos at $6.5-7.5 \mathrm{hr}$ of development (Fig. 2B,G). In thoracic segments, labeling was seen in cells presumably belonging to basiconical sensilla [les3 (13) and $v^{\prime}$ es3 (v'3)]. In abdominal segments at the same stage, very weak expression was seen in cells belonging to des2 (arrow in Fig. 2G). In T2-T3 of an 8- to 9-hr embryo (Fig. 2D,H), strong BarH1/BarH2 expression in the Keilin's organ and weak BarH1/BarH2 expression in a dorsal simple es organ was evident. In A1-A7 at the same stage, the expression in the cells of des2 (d2) be- 


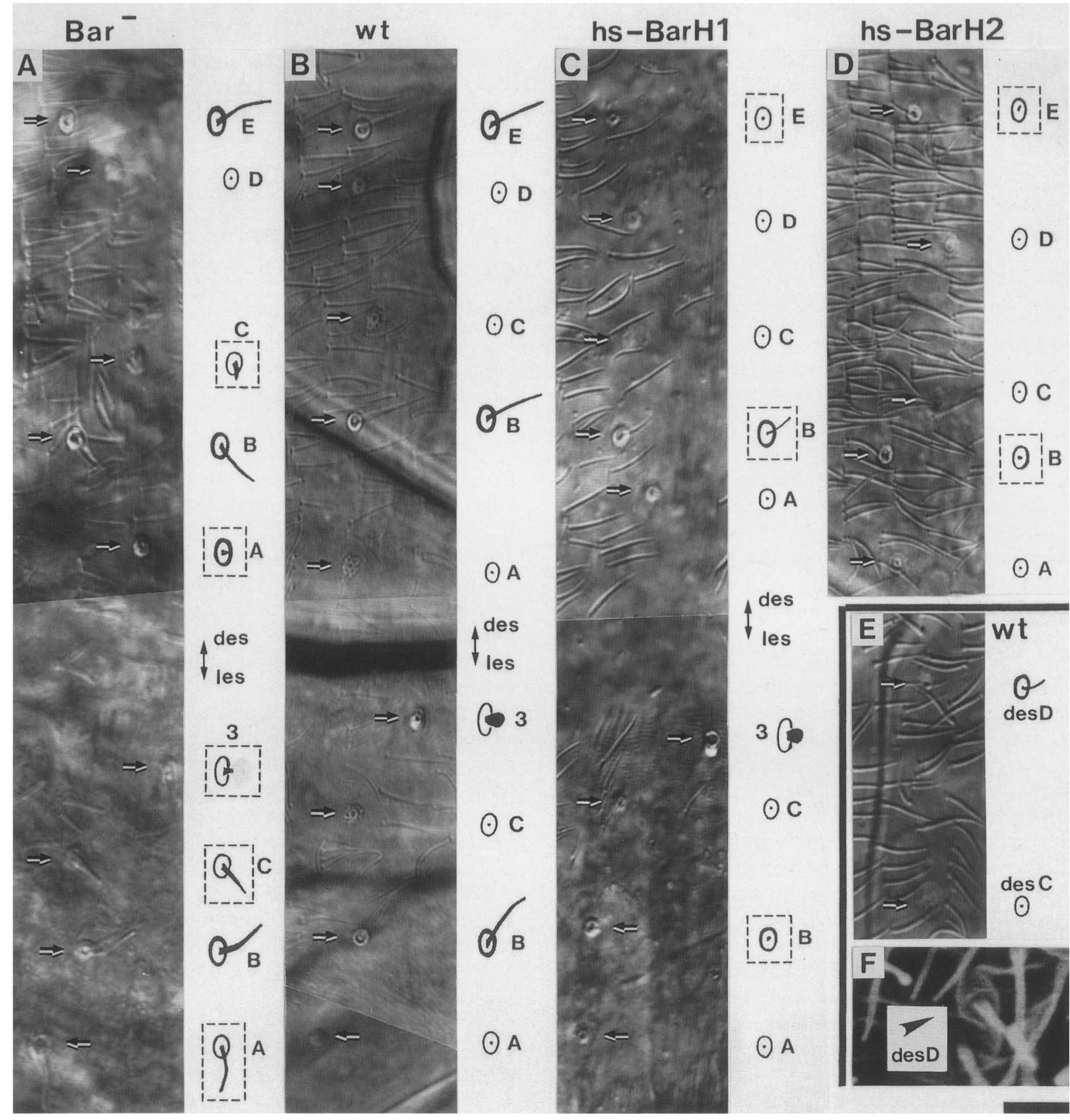

Figure 8. Induction of morphological changes of sensory organs by the altered expression of BarH1 and BarH2 genes. (A-C) Lateral and dorsal regions of a thoracic segment $(\mathrm{T} 3)$ of the first-instar larvae. $(A) D f(1) B^{263-20} ;(B)$ wild type; $(C)$ a wild-type larva in which the exogenous $h s-B a r H 1$ fusion gene was heat induced at $11 \mathrm{hr}$ after fertilization; $(D)$ dorsal region of a thoracic segment (T3) of a first-instar larva, in which the exogenous hs-BarH2 fusion gene was heat induced at $1 \mathrm{l} \mathrm{hr}$ after fertilization. All photographs were taken using Nomarski optics; morphological features of es organs are schematically shown in the right margin of each picture. Dotted rectangles indicate transformed es organs. $(E)$ Dorsal region of an abdominal segment of a wild-type first-instar larva. Note that the des $\mathrm{D}$ campaniform has a bristle with a length about one-quarter that of a typical trichoid sensillum. (F) A scanning electron micrograph of the abdominal desD of the wild-type first-instar larva Bar, $10 \mu \mathrm{m}$ in $A-E$ and $3 \mu \mathrm{m}$ in $F$.

comes prominent, and very weak BarH1/BarH2 expression, probably corresponding to $\mathrm{v}^{\prime} \mathrm{es} 2\left(\mathrm{v}^{\prime} 2\right)$, could be seen in the ventrolateral region (arrowhead in Fig. $2 \mathrm{H}$ ). Staining of most simple es organs was apparent in 10- to $11-\mathrm{hr}$ embryos (Fig. 2E,F,I,J).

A previous experiment has shown that DNA replica- tion of most embryonic sensory organs occurs in 6- to 8-hr embryos (Bodmer et al. 1989). In fact, almost all of trichogen-tormogen and dorsal neuron-thecogen precursors finish their DNA replication by $8 \mathrm{hr}$ following egg fertilization. DNA replication of the lateral and ventral neuron-thecogen precursors probably occurs in 8- to 9 -hr 
embryos. Neural antigen expression is initially apparent in neurons of simple es organs at 11-12 hr of development (Hartenstein 1988). The expression of BarH1/ $\mathrm{BarH} 2$ would thus appear to occur in simple es organs in thoracic and abdominal segments immediately following the second cycle of DNA replication of es primodial cells and shortly before expression of the neural antigen.

\section{Discussion}

The expression of two closely related homeo box genes, BarH1 and BarH2, was examined in Drosophila embryos using antibodies specific to their gene products. Bar homeo domain proteins were coexpressed in a limited number of cells of the CNS and es organs in the PNS. In es organs, BarH1 and BarH2 proteins were found to be expressed at the final stage of development and to be essential to the fate determination of these organs.

\section{Sensillum formation and Bar homeo box genes}

Although their morphologies clearly differ, trichoid and campaniform-like sensilla are a pair of sibling, simple es organs in thoracic and abdominal segments in Drosophila embryos. Each consists of four cells, derivatives of two cycles of DNA replication of a single progenitor cell, the first precursor cell (see Fig. 7). One of the second precursor cells produces a neuron and a thecogen, whereas the other produces a trichogen and a tormogen (Bodmer et al. 1989). The neural antigen in these organs is initially expressed in 11- to 12 -hr embryos (Hartenstein 1988), whereas axon projection and dendrite innervation are complete for the most part at $12-14 \mathrm{hr}$ after egg fertilization. Massive cuticle secretion occurs thereafter. No significant difference was noted between trichoid and campaniform-like sensilla of 12- to $14-\mathrm{hr}$ embryos using various cell-type-specific markers, including neuron-specific antibodies (see Fig. 3F), suggesting that these simple es organs differentiate in an almost identical fashion and acquire their identity at relatively later or final stages of development. Our finding of the conversion from campaniform-like sensilla to trichoid sensilla by the introduction of a small deficiency in the Bar region (Fig. 5A) shows that the deleted region may contain selector genes responsible for subtype determination.

Our previous study indicated that the region deleted in $D f(1) B^{263-20}$ is $210 \mathrm{~kb}$ in length and contains at least five transcriptional units, two corresponding to BarH1 and BarH2 (Higashijima et al. 1992). The remaining are the putative forked gene (X1), a possible transposon (T1/T2), and an unknown gene, X2, expressing throughout development (Fig. 5A). No conversion between campaniformlike and trichoid sensilla could be found in embryos with a null mutation of the forked gene (S. Higashijima et al., unpubl.). T1/T2 also may not be related to the morphogenesis of es organs, because the T1 region of $\mathrm{T} 1 / \mathrm{T} 2$ was deleted in the $B$ mutant, exhibiting normal sensillum structures (Higashijima et al. 1992).

Both BarH1 and BarH2 proteins have homeo domains and are coexpressed in the nuclei of cells of es organs.
This would be an indication of the regulatory function of dual Bar homeo domain proteins as transcriptional regulators, as has been suggested for other homeo domaincontaining proteins causing homeotic transformation (for review, see Levine and Hoey 1988). The expression of BarH1/BarH2 proteins in thecogens and neurons of simple es organs becomes apparent at relatively later developmental stages (Fig. 7), and subtype selection was found to be quite closely related to the level of $\mathrm{BarH1} / \mathrm{BarH} 2$ expression in es neurons. All of these findings are consistent with the notion that dual Bar homeo box genes are responsible for sensillum determination. The altered expression of BarH1 and/or BarH2 could bring about conversion between trichoid and campaniform-like sensilla.

A similar example of sensillum subtype conversion is the mutation of the cut locus. In the absence of cut gene activity, es organs are transformed to ch organs. As with BarH1 and BarH2, the cut gene encodes a homeo domain-containing protein, which is expressed in all cells of es organs, but not in any cell of a ch organ (see Fig. 7). There is thus a fundamental difference in expression between cut and BarH1/BarH2. The cut protein is expressed only in one of two intercompatible organs, whereas BarH1/BarH2 proteins are expressed in both, indicating that, in contrast to most homeotic genes including cut, BarH1 and/or BarH2 function not as digital switches for differentiation but as analog switches. This would also partially explain the presence of various types of intermediates or hybrids for trichoid and campaniform-like sensilla, found in Bar-region deficient flies, flies that underwent reverse morphological change, and even wild-type flies.

Larval campaniform-like sensilla may form a sensillum group different from that of the classical campaniform sensilla (Hodgkin and Bryant 1978). Our scanning electron micrographs in Figure 6, J-M, raise the possibility that the larval campaniform-like sensilla may be a kind of trichoid sensilla with extremely truncated hairs. If so, a straightforward interpretation of our results is that it is a quantitative differentiative change in the hairforming (trichogen) cell that dual Bar homeo box genes, $\mathrm{BarH1}$ and $\mathrm{BarH2}$, control.

Cuticle structure change induced by BarH1 (and/or $B a r H 2)$ overexpression also provides a clue as to time of sensillum subtype determination under the control of BarH1 and/or BarH2, because it may take place without any assistance from endogenous dual Bar homeo box genes. Immunostaining showed that the overexpressed BarH1 protein remained for $\sim 2 \mathrm{hr}$ following heat treatment (data not shown). That 10- to 11 -hr heat treatment affected reverse morphological change most strongly indicates that $\mathrm{BarH1}$ (or $\mathrm{BarH} 2$ ) protein possibly determines the sensillum subtype at $\sim 10-13 \mathrm{hr}$ following egg fertilization. This was supported further by immunostaining experiments in wild-type embryos. The expression of $\mathrm{BarH1} / \mathrm{BarH} 2$ proteins in simple es organs was initially seen at $\sim 10 \mathrm{hr}$ of development; it reached a considerable level at $\sim 11-12 \mathrm{hr}$, and persisted until at least $14 \mathrm{hr}$ at which time immunostaining became diffi- 
cult owing to cuticle formation. Heat treatment of 11 - to -13-hr embryos caused only partial morphological change, suggesting that the fate of most sensilla has already been determined to a certain degree at least at 12 $\mathrm{hr}$ following egg fertilization.

The expression of BarH1/BarH2 proteins in simple es organs was restricted to neurons and internal support cells, thecogens, whereas sensillum morphology change was seen in cuticle structures secreted from outer support cells in which BarH1/BarH2 were not expressed (Fig. 7). Thus, neurons, thecogens, or both may play key roles in determining sensillum subtype, possibly by cellcell interactions among the postmitotic sensillum cells. This finding is of particular importance, because traditionally the emphasis regarding sensillum cell determination has always been on cell lineage and cell autonomy (Bodmer et al. 1989). The simple es organ consists of only four cells, easily identifiable with various cell typespecific markers. It may be a good model system for analyzing molecular mechanisms of cell-cell communication possibly involved in nervous system differentiation.

\section{Relation between $\mathrm{BarH1}$ and $\mathrm{BarH} 2$}

Possibly no fundamental differences in biological function exist between BarH1 and BarH2 proteins, at least with respect to es cell formation. They commonly share a homeo domain very similar in sequence and appear capable of recognizing an identical set of DNA sequences as targets (Higashijima et al. 1992). BarH1 and BarH2 are coexpressed equally in $>1000$ embryonic cells, most being related to the PNS or the CNS (Fig. 1B-G). The overexpression of either $\mathrm{BarH} 1$ or $\mathrm{BarH} 2$ results in conversion from trichoid sensilla to campaniform-like sensilla in thoracic and abdominal segments, whereas their deletion causes the opposite homeotic changes (see Fig. 7). $\mathrm{BarH} 1$ and $\mathrm{BarH} 2$ proteins would thus appear capable of functioning as similar homeotic selectors. The finding that the BarH2 null mutation is not lethal and induces no appreciable transformation in sensillum structure is consistent with the notion that endogenous BarH1 and $\mathrm{BarH} 2$ genes are redundant in their functions. This is so because the effect of inactivation of one of the two functionally related genes (redundant genes) should be compensated for by the other, as shown by yeast ras-related genes (Kataoka et al. 1984; Tatchell et al. 1984). At present, whether the endogenous BarH2 functions as a complete substitute of the endogenous $\mathrm{BarH1}$ is a point yet unclarified owing to the absence of the $\mathrm{BarH1}{ }^{-}$ $\mathrm{BarH}^{+}$mutant. However, our recent finding that Keilin's organs in $\operatorname{In}(1) B^{M 2}$ flies are immunologically BarH1- BarH2 $^{+}$but normal in sensillum shape (unpubl.) would support the possibility of such a function.

BarH1 and BarH2 are strictly coexpressed. No embryonic cell, in which only one of the two is expressed, has so far been detected. This strict coexpression may be the result of the presence of common enhancers in the $80-\mathrm{kb}$ region flanked by $\mathrm{BarH1}$ and BarH2. In the embryonic CNS of $\operatorname{In}(1) B^{M 2}$, whose breakpoint is located within this $80-\mathrm{kb}$ region (Fig. 5A), BarH1 protein expression is restricted to three mid-line cells in each segment, whereas that of the $\mathrm{BarH} 2$ protein occurs exclusively in the remaining CNS cells that are positive to both $\mathrm{BarH1}$ and $\mathrm{BarH} 2$ in the case of wild type (S. Higashijima et al., unpubl.).

\section{Materials and methods}

Fly strains and genetics

$D f(1) B^{263-20}$ (Sutton 1943) was obtained from Mid-America Drosophila Stock Center (Bowling Green State University, Bowling Green, OH). Enhancer trap lines, A1-2nd-29 (Hartenstein and Posakony 1990) and ryxho25 (Hama et al. 1990) were obtained from Y.N. Jan (University of California, San Francisco) and C. Hama (National Institute of Neuroscience, Tokyo), respectively. The line Q6, in which lacZ is expressed in all cells of the embryonic PNS, was generated in our laboratory ( $M$. Sone, unpubl.). yellow was introduced into $D f(1) B^{263-20}$ to identify larvae hemizygous for this deficiency. $y^{-}$larvae are distinguishable from the wild-type larvae by their yellow mouthhooks. Virgin females of $D f(1) B^{263-20} y / \operatorname{In}(1) s c^{7}, \operatorname{In}(1) A M s c^{7} c a r$ $\left(\mathrm{y}^{+}\right.$first chromosomal balancer) were crossed to wild-type males. Larvae having yellow mouth-hooks and constituting about one-quarter of the progeny, died 1-3 days after hatching, indicating that $D f(1) B^{263-20}$ is lethal in an early larval stage. A $B a r H 2$ null mutant $(D f(1) B H 2)$ was generated by recombination between $B$, having a $B a r H 1$ duplication, and $D f(1) B^{263-20}$, including forked, as shown schematically in Figure 5B. One forked fly found in 20,000 male progeny was verified to be $\mathrm{BarH}^{-} \mathrm{BarH1}^{+}$by Southern blotting and immunostaining. First-instar larvae homozygous and/or hemizygous for $f^{S H}$, an extreme forked allele isolated by us [S. Higashijima et al., unpubl.), were used to examine the effect of the forked mutation on larval cuticle structures. Under a forked ${ }^{-}$condition, only subtle changes such as slightly shortened hairs and increased susceptibility to Hoyer's solution were observed, but no campaniform-trichoid transformation could be observed.

\section{Preparation of antibodies}

The S12 antibody was prepared as follows. A cDNA fragment encoding the carboxy-terminal three-fifths of the BarH1 protein (amino acid positions 250-543) was inserted into the pET3a vector (Rosenberg et al. 1987), and the T7-gene-10-BarH1 fusion protein was overexpressed in E. coli cells (Studier and Moffatt 1986). After cells were collected, suspended in $10 \mathrm{~mm}$ phosphate buffer ( $\mathrm{pH} 7.2)$, and disrupted by sonication, the supernatant fluid of a centrifugation at $10,000 \mathrm{~g}$ for $20 \mathrm{~min}$ was loaded on a DE52 (Whatmann) column. After washing with $0.3 \mathrm{M}$ $\mathrm{NaCl}$, the fused $\mathrm{BarH1}$ protein, whose purity was $80-90 \%$, was eluted by $0.5 \mathrm{M} \mathrm{NaCl}$. Dialyzed protein preparations were combined and used for immunization of rabbits. The antiserum thus obtained was affinity purified. The extract of E. coli cells expressing the fused $\mathrm{BarH} 1$ protein was size-fractionated by SDSpolyacrylamide gel electrophoresis and transferred to PVDV filter (Millipore), and a narrow strip including the fused $\mathrm{BarH1}$ was removed. After blocking, this strip was incubated with the anti-BarH1 antiserum. The bound antibody fraction was eluted by washing the strip with $0.2 \mathrm{M}$ glycine (pH 2.5), quickly neutralized with Tris base, and dialyzed against PBS.

The Y2 antibody was prepared as follows. After a cDNA fragment encoding the $\mathrm{BarH} 2$ protein lamino acid positions 196640) was cloned into pUC19, the fused $\mathrm{BarH} 2$ protein was overexpressed in E. coli cells, and partially purified by SDS-poly- 
acrylamide gel (purity, $\sim 90 \%$ ). The combined preparations of the fused BarH2 protein were used for immunization. The resultant antiserum was affinity purified as described above.

The S1-1 antibody was obtained by absorption of the S12 antibody with the extract of $E$. coli cells expressing the fused $\mathrm{BarH2}$ protein.

\section{Immunohistochemistry and in situ hybridization}

Whole-mount staining of embryos was performed essentially as described by Thomas et al. (1987), except the Vecta-stain ABCHRP kit (Vector) was used for signal detection. Rabbit anti-HRP antibody (Cappel) was affinity purified by the HRP protein (Sigma fraction VI), and used at $1: 100$ dilution. mAb22C10 (1:4) was kindly supplied by S. Fujita (Mitsubishi-Kasei Institute of Life Sciences, Tokyo), whereas purified rabbit anti-lac $Z$ antibody $(1: 2000$; Cappel), mouse monoclonal anti-lac $Z$ antibody $(1: 200$; Promega $)$, and biotinylated anti-rabbit or antimouse antibodies (1:500; Vector) were commercial products. ABC-HRP reaction was carried out according to the manufacturer's protocol. For signal intensification, $0.4 \mathrm{mg} / \mathrm{ml}$ of $\mathrm{NiCl}_{2}$ was added to the standard $3,3^{\prime}$-diaminobenzidine (DAB) color development reaction $10.5 \mathrm{mg} / \mathrm{ml}$ of DAB and $0.003 \%$ of $\mathrm{H}_{2} \mathrm{O}_{2}$ in PBS!.

Double staining was conducted as follows. Following the first-cycle staining, which is described above (black color development), the second-cycle staining was conducted without adding $\mathrm{NiCl}_{2}$ during color development (brown color development).

Samples were mounted on Canada balsam/xylene after dehydration in series of ethanol or mounted directly on $90 \%$ glycerol and flattened by the weight of the coverslip. Some samples were dissected longitudinally in $90 \%$ glycerol and mounted on the same solution.

Whole-mount in situ hybridizations were performed essentially according to Tautz and Pfeifle (1989), except for the usage of a digoxigenin-labeled antisense RNA probe. For probe labeling, nonhomologous regions were selected. In the case of $\mathrm{BarH1}$, a 0.6-kb NotI-EcoRI fragment of one cDNA clone (cl-d7) was used. The NotI site corresponds to $414-416$ amino acid positions of the predicted BarH1 protein (Kojima et al. 1991), and the EcoRI site exists at the $3^{\prime}$-noncoding region in this cDNA clone. In the case of $\mathrm{BarH2}$, a 0.9-kb EcoRI-Sall fragment derived from the BarH2 $3^{\prime}$-noncoding region was used, [fragment 1 in Fig. 1 of Higashijima et al. (1992)].

\section{Cuticle observation}

Cuticle samples of first-instar larvae were prepared as described by Wieschaus and Nüsslein-Volhard (1986). After being mounted in Hoyer's solution, samples were squashed by a coverslip and photographed immediately. In particular, in the case of larvae homozygous or hemizygous for forked, photographs were taken within $2 \mathrm{~min}$, because of their high susceptibility to Hoyer's solution. Larvae for scanning electron microscopy were prepared as described by Grodowitz et al. (1982). Larvae were collected, washed with water, and placed in supper skipper /kerosene $17 \%$, glacial acetic acid $11 \%, 95 \%$ ethanol $50 \%$, 2-methyl-1-propanol $17 \%$, and dioxane 5\%) for 15-30 sec. After being rinsed twice in Carl's fixative (95\% ethanol 28, 20\% paraformaldehyde 22 , glacial acetic acid 4 , and water 46 ), samples were fixed in the same solution for 12-24 hr and gradually dehydrated by $30-100 \%$ ethanol. Samples were critical-pointdried with liquid carbon dioxide and coated by platinum-palladium.

\section{Heat-shock treatment}

Transgenic flies having the BarH1 minigene associated with a heat-shock promoter have been described previously (Kojima et al. 1991). Two independent viable homozygous lines, B27 and $B H 102$, were used in which the BarH1 minigene is located on the second and first chromosomes, respectively. Similar transgenic flies (hs-BarH2-49) with the BarH2 minigene have also been constructed (details will be described elsewhere). Transgenic embryos were heat-shocked at $37^{\circ} \mathrm{C}$ for $15-20 \mathrm{~min}$, and morphological changes found in first-instar larvae were examined. No morphological changes in es organs were detected on heat treatment in the case of wild-type larvae. For the examination of heat shock effects under a $D f(1) B^{263-20}$ background, virgin females $D f(1) B^{263-20} \mathrm{y} / \operatorname{In}(1) s c^{7}, \operatorname{In}(1) A M s c^{7}$ car were crossed to $B 27$ males, and effects on resultant yellow larvae were examined.

\section{Acknowledgments}

We thank Y.N. Jan and C. Hama for Drosophila strains A1-2nd29 and ryxho25, respectively. We also thank R. Ueda, T. Uemura, and T. Kojima for helpful discussions. This work was supported in part by grants from the Ministry of Education, Science, and Culture of Japan to K.S.

The publication costs of this article were defrayed in part by payment of page charges. This article must therefore be hereby marked "advertisement" in accordance with 18 USC section 1734 solely to indicate this fact.

\section{References}

Blochlinger, K., R. Bodmer, J.W. Jack, L.Y. Jan, and Y.N. Jan. 1988. Primary structure and expression of a product from cut, a locus involved in specifying sensory organ identity in Drosophila. Nature 333: 629-635.

Blochlinger, K., R. Bodmer, L.Y. Jan, and Y.N. Jan. 1990. Patterns of expression of Cut, a protein required for external sensory organ development in wild-type and cut mutant Drosophila embryos. Genes \& Dev. 4: 1322-1331.

Blochlinger, K., L.Y. Jan, and Y.N. Jan. 1991. Transformation of sensory organ identity by ectopic expression of Cut in Drosophila. Genes \& Dev. 5: 1124-1135.

Bodmer, R. and Y.N. Jan. 1987. Morphological differentiation of the embryonic peripheral neurons in Drosophila. Wilhelm Roux's Arch. Dev. Biol. 196: 69-77.

Bodmer, R., S. Barbel, S. Shepherd, J.W. Jack, L.Y. Jan, and Y.N. Jan. 1987. Transformation of sensory organs by mutation of the cut locus of D. melanogaster. Cell 51: 293-307.

Bodmer, R., R. Carretto, and Y.N. Jan. 1989. Neurogenesis of the peripheral nervous system in Drosophila embryos: DNA replication patterns and cell lineages. Neuron 3: 21-32.

Campos-Ortega, I.A. and V. Hartenstein. 1985. The embryonic development of Drosophila melanogaster. Springer-Verlag, Berlin, Germany.

Dambly-Chaudiere, C. and A. Ghysen. 1986. The sense organs in the Drosophila larva and their relation to the embryonic pattern of sensory neurons. Wilhelm Roux's Arch. Dev. Biol. 195: 222-228.

Doe, C.Q., Q. Chu-LaGraff, D.M. Wright, and M.P. Scott. 1991. The prospero gene specifies cell fates in the Drosophila central nervous system. Cell 65: 451-464.

Fuiita, S., S.L. Zipursky, S. Benzer, A. Ferrus, and S.L. Shotwell. 1982. Monoclonal antibodies against the Drosophila nervous system. Proc. Natl. Acad. Sci. 79: 7929-7933. 
Ghysen, A., C. Dambly-Chaudiere, E. Aceeves, L.Y. Jan, and Y.N. Jan. 1986. Sensory neurons and peripheral pathways in Drosophila embryos. Wilhelm Roux's Arch. Dev. Biol. 195: 281-289.

Grodowitz, V.A., J. Krchma, and A.B. Broce. 1982. A method for preparing soft bodied larval diptera for scanning electron microscopy. I. Kans. Entomol. Soc. 55: 751-753.

Hama, C., Z. Ali, and T.B. Kornberg. 1990. Region-specific recombination and expression are directed by portions of the Drosophila engrailed promoter. Genes \& Dev. 4: 10791093.

Hartenstein, V. 1988. Development of Drosophila larval sensory organs: Spatiotemporal pattern of sensory neurons, peripheral axonal pathways and sensilla differentiation. Development 102: 869-886.

Hartenstein, V. and J.W. Posakony. 1990. A dual function of the Notch gene in Drosophila sensilla development. Dev. Biol. 142: $13-30$.

Higashijima, S., T. Kojima, T. Michiue, S. Ishimaru, Y. Emori, and K. Saigo. 1992. Dual Bar homeo box genes of Drosophila required in two photoreceptor cells, R1 and R6, and primary pigment cells for normal eye development. Genes \& Dev. 6: $50-60$.

Hodgkin, N.M. and P.J. Bryant. 1978. Scanning electron microscopy of the adult of Drosophila melanogaster. In The genetics and biology of Drosophila (ed. M. Ashburner and T.R.F. Wright), vol. 2c, pp. 337-358. Academic Press, London, UK.

Jan, L.Y. and Y.N. Jan. 1982. Antibodies to horseradish peroxidase as specific neuronal markers in Drosophila and in grasshopper embryos. Proc. Natl. Acad. Sci. 72: 2700-2704.

Kataoka, T., S. Powers, C. McGill, O. Fasano, J. Strathern, J. Broach, and M. Wigler. 1984. Genetic analysis of yeast RAS1 and RAS2 genes. Cell 37: 437-445.

Kojima, T., S. Ishimaru, S. Higashijima, E. Takayama, H. Akimaru, M. Sone, Y. Emori, and K. Saigo. 1991. Identification of a different-type homeobox gene, BarH1, possibly causing Bar $(B)$ and $O m(1 D)$ mutations in Drosophila. Proc. Natl. Acad. Sci. 88: 4343-4347.

Levine, M. and T. Hoey. 1988. Homeobox proteins as sequencespecific transcription factors. Cell 55: 537-540.

Matsuzaki, F., K. Koizumi, C. Hamma, T. Yoshioka, and Y. Nabeshima. 1992. Cloning of the Drosophila prospero gene and its expression in ganglion mother cells. Biochem. Biophys. Res. Commun. 182: 1326-1332.

McIver, S.B. 1985. Mechanoreception. In Comprehensive insect physiology, biochemistry and pharmacology (ed. G.A. Kerkut and L.I. Gilbert), vol. 6, pp. 71-132. Pergamon Press, New York.

Rosenberg, A.I., B.N. Lade, D. Chui, S.-W. Lin, J.J. Dunn, and F.W. Studier. 1987. Vectors for selective expression of cloned DNAs by T7 RNA polymerase. Gene 56: 125-135.

Studier, F.W. and B.A. Moffatt. 1986. Use of bacteriophage T7 RNA polymerase to direct selective high-level expression of cloned genes. I. Mol. Biol. 189: 113-130.

Sturtevant, A.H. 1925. The effects of unequal crossing-over at the Bar locus in Drosophila. Genetics 10: 117-147.

Sutton, E. 1943. Bar eye in Drosophila melanogaster: A cytological analysis of some mutations and reverse mutations. Genetics 28: 97-107.

Tatchell, K., D.T. Chaleff, D. DeFeo-Jones, and E.M. Scolnick. 1984. Requirement of either of a pair of ras-related genes of Saccharomyces cerevisiae for spore viability. Nature 309: 523-527.

Tautz, D. and D. Pfeifle. 1989. A nonredioactive in situ hybridization method for the localization of specific RNAs in Drosophila reveals a tranlational control of segmentation gene hunchback. Chromosoma 98: 81-85.

Thomas, J.B., S.T. Crews, and C.S. Goodman. 1987. Molecular genetics of single-minded locus: A gene involved in the development of Drosophila nervous system. Cell 52: 133-141.

Tsubota, S.I., D. Rosenberg, H. Szostak, D. Rubin, and P. Shedl. 1989. The cloning of the Bar region and $B$ breakpoints in Drosophila melanogaster: Evidence for a transposon-induced rearrangement. Genetics 122: 97-108.

Uemura, T., S. Shepherd, L. Ackermann, L.Y. Jan, and Y.N. Jan. 1989. numb, a gene required in determination of cell fate during sensory organ formation in Drosophila embryos. Cell 58: $349-360$.

Vaessin, H., E. Grell, E. Wolff, E. Bier, L.Y. Jan, and Y.N. Jan. 1991. prospero is expressed in neuronal precursors and encodes a nuclear protein that is involved in the control of axonal outgrowth in Drosophila. Cell 67: 941-953.

Wieschaus, E. and C. Nüsslein-Volhard. 1986. Looking at embryos. In Drosophila: A practical approach (ed. D.B. Roberts|, pp. 199-227. IRL Press, Oxford, UK.

Zaharuk, R.Y 1985. Antennae and sensilla. In Comprehensive insect physiology, biochemistry and pharmacology (ed. G.A. Kerkut and L.I. Gilbert), vol. 6, pp. 1-69. Pergamon Press, New York.

Zipursky, S.L., T.R. Venkatesh, D.B. Teplow, and S. Benzer. 1984. Neuronal development in the Drosophila retina: Monoclonal antibodies as molecular probes. Cell 36: 15-26. 


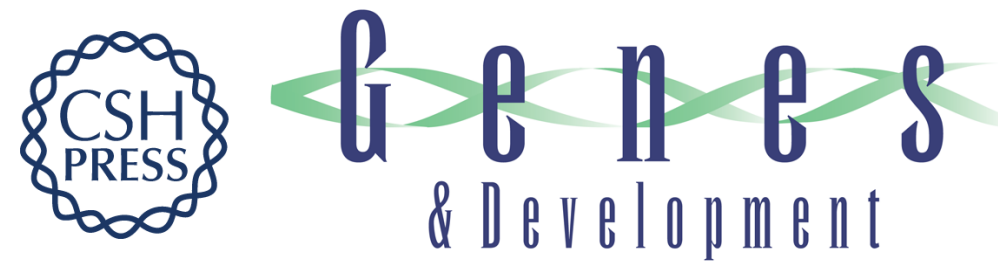

\section{Subtype determination of Drosophila embryonic external sensory organs by redundant homeo box genes BarH1 and BarH2.}

$S$ Higashijima, T Michiue, Y Emori, et al.

Genes Dev. 1992, 6:

Access the most recent version at doi:10.1101/gad.6.6.1005

References This article cites 31 articles, 9 of which can be accessed free at:

http://genesdev.cshlp.org/content/6/6/1005.full.html\#ref-list-1

License

Email Alerting

Service

Receive free email alerts when new articles cite this article - sign up in the box at the top right corner of the article or click here.

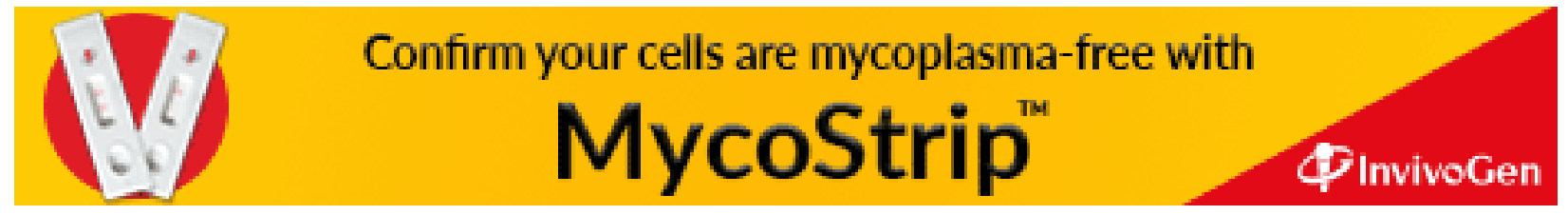

